\title{
Oriented nanofibrous membranes for tissue engineering applications: Electrospinning with secondary field control.
}

Jochen Walser ${ }^{1,2 *}$, Stephen J. Ferguson ${ }^{1,2}$

${ }^{1}$ ETH Zurich, Institute for Biomechanics, Zurich, $\mathrm{CH}$

${ }^{2}$ Collaborative Research Partner of AO Foundation, Davos, $\mathrm{CH}$

*Corresponding Author:

Jochen Walser

Address:

Institute for Biomechanics

HPP 014

Hönggerbergring 64

$\mathrm{CH}-8093$ Zürich

Switzerland

Tel: + $410446334013 \quad$ Fax: + 410446331453

E-mail: jowalser@ethz.ch

Prof. Dr. Stephen J. Ferguson

Institute for Biomechanics

HCl E 355.2

Vladimir-Prelog-Weg 1-5/10

$\mathrm{CH}-8093$ Zurich

Switzerland

Phone: + $41446339330 \quad$ Fax: + 410446331453

E-Mail: sferguson@ethz.ch 


\section{Abstract}

Electrospinning is an electrical field driven method to produce polymer fibre mats or membranes by deposition of a charged polymer jet onto a grounded collector and has shown to be very promising for tissue engineering applications. Fibre alignment within these mats is usually achieved by a fast collector movement, which is not feasible for all collector geometries, such as small diameter tubes or free-form moulds. The aim of this study was to evaluate the use of charged deflector plates to apply a dynamic, alternating electrical field perpendicular to the spinning direction, in order to directly control the fibre trajectory.

Different field signal types, deflector plate voltages $\left(\mathrm{V}_{\mathrm{pp}}=12 \mathrm{kV}, 16 \mathrm{kV}\right)$ and deflection frequency ranges $\left(f_{d}=0.5 . .160 \mathrm{~Hz}\right)$ have been investigated. 210 poly $(\varepsilon)$ caprolactone $(\mathrm{PCL})$ membranes were electrospun. SEM images, taken from the centre as well as from the side regions of each membrane were analysed using ImageJ. Main fibre diameter and orientation, as well as the degree of fibre alignment, were calculated. Furthermore, strips were cut from a subset of the spun scaffolds, tested for their tensile properties in relation to the relevant fibre ratio parallel $\left(P_{\| I}\right)$ to the test direction. Analysis showed no significant difference in alignment between membranes produced with the different signal types in the high frequency range. A higher deflector plate voltage amplitude resulted in a smaller variance and hence a better fibre alignment. Although the estimated fibre deposition rate suggests otherwise, the best alignment was observed in a low deflection frequency range from 2 to $10 \mathrm{~Hz}$. Mean main fibre direction was $87 \pm 18$ degrees, relative to the deflection axis, while fibre alignment seems to have only a minor effect on the average fibre diameter. 
Young's modulus and yield stress increased with the ratio of the parallel fibre component from min. 2.0 to $\max$. 9.3 $\mathrm{MPa}$ and 0.1 to $0.7 \mathrm{MPa}$, respectively. The feasibility of the described method to achieve fibre alignment could be demonstrated. Low frequency deflection seems to give the natural whipping effect of the polymer jet a preferred direction and leads to better alignment than direct high frequency fibre deposition attempts. However, the main fibre direction is not aligned with the deflection axis, but rather consistently perpendicular to it, which is also reflected in the tensile properties of spun samples.

Keywords: electrospinning, auxiliary electrodes, deflector plates, caprolactone, fibre alignment. 


\section{Introduction}

Electrospinning is an electrical field driven method to produce polymer fibre mats or membranes by deposition of a charged polymer jet onto a grounded collector and has shown to be very promising for tissue engineering applications (Bhardwaj and Kundu, 2010; George, 2011; Nisbet et al., 2009; Pham et al., 2006). In more detail, a polymer solution is charged using a potential of $20-30 \mathrm{kV}$ at the needle tip of a syringe. In this range, electrostatic repulsion exceeds surface tension of the polymer solution in the droplet at the tip and a small polymer jet follows the electric field lines and is driven towards a collector, which is connected to ground potential, while the solvent evaporates on its way to the collector. The outcome of the spinning process is governed by parameters like gap distance, electrode potential, polymer solution, feed rate, but also by collector geometry and the electrospinning environment (temperature, humidity etc.). Beside the described basic process, many different approaches have been made to either tune the mechanical properties or the morphology of the membranes. However, changes in morphology also lead to changes in the mechanical properties of the construct and vice versa. An increase of pore size leads to a decrease of the tensile strength, while increasing fibre alignment is accompanied by a decrease in pore size. Nevertheless, alignment of the electrospun fibres is a popular approach to tune or increase the tensile stiffness and strength of spun membranes in preferential fibre direction, to provide a specified degree of mechanical anisotropy and/or mechanical property enhancement, and is usually achieved by two different techniques. These techniques include either a fast collector 
movement, such as spinning onto a rotating mandrel (Baji et al., 2010; Courtney et al., 2006; Kumbar et al., 2008; Li et al., 2007; Milleret et al., 2011; Nerurkar et al., 2007) or mounting the collector plate onto moving $x-y$ stage (Kim, 2008), or using special collector geometries, such as two or more collector blades (Kumbar et al., 2008; Secasanu et al., 2009; Teo and Ramakrishna, 2005). However, due to a fibre deposition rate of usually several meters per second, a sufficiently fast collector movement, on the order of $10 \mathrm{~m} / \mathrm{s}$, is not feasible for all collector geometries, such as small diameter tubes or free-form moulds, due to their shape and also their inertia. Moreover, the two-blade technique is bound to the geometry of the blades, or rather the gap space in-between, while furthermore only a small fraction of the deposited fibres are actually bridging the two blades as parallel-aligned fibres and contributing to the creation of the desired fibre structure.

There have been a few reports of the use of auxiliary electrodes and / or deflector plates to better condition the primary electric field or also achieve a static deflection (George, 2011; Heikkilä et al., 2007; Nurfaizey et al., 2011; Nurfaizey et al., 2014). Arras et al. (2012) used deflector plates to dynamically direct the spot of fibre deposition on a rotating mandrel and to enhance fibre alignment on the rotating mandrel. Grasl et al. (2013) reported the use of deflector plates only to achieve fibre alignment on a static collector by applying a high frequency $(30 \mathrm{~Hz}-60 \mathrm{~Hz})$ square wave signal to generate a secondary field. The aim of the present study was to further evaluate the latter method to achieve fibre alignment, i.e. the use of charged deflector plates to apply an alternating, dynamic electrical field perpendicular to the spinning direction. Specifically, we investigated the resulting alignment and mechanical 
properties across a broad range of deflection potentials and frequencies, with different deflection signal types.

Hypothesis: Achieving fibre alignment by dynamic polymer jet deflection is possible and will be aligned with the deflection axis. An optimal frequency can be found, but is probably specific for the particular setup and spinning parameters. Furthermore, the degree of fibre alignment should be reflected in the tensile properties of the thus spun membranes.

\section{Methods and Materials}

\subsection{Electrospinning setup design}

The geometrical parameters of the setup, as well as the applied potentials, were chosen based on 3D finite element simulations of the resulting electric fields (Comsol Multiphysics, Comsol Inc.) (Appendix A). The goal of the FEM model was to define initial apparatus dimensions and spinning parameters (deflector plate size, distances and potentials), which would theoretically result in a maximum jet deflection without fibre being deposited on the deflector plates, i.e. no field line starting from the needle tip would reach the deflector plates, given the limitations of a maximum deflector potential of $\pm 10 \mathrm{kV}$. The geometric parameters as well as the applied potentials served as input or boundary conditions respectively. The output of the model was the electric field strength (Appendix A). The polymer jet was not part of the model, hence polymer-field-interactions were not considered in the simulation. The result of the simulation was the setup as described below and shown in Figure 1.

\subsection{Electrospinning}


Polymer membranes were spun from 8wt\% Polycaprolactone $(\mathrm{PCL}, \mathrm{Mw}=80000$, Sigma-Aldrich, St. Louis, USA) dissolved in a 1:6 mixture of methanol (MeOH) and chloroform $(\mathrm{CHCl} 3)$ using the electrospinning setup showed in Figure 1. The main spinning process was driven by a Heinzinger LNC 30000-2 high voltage power supply at a constant needle (18G) tip voltage of $26.5 \mathrm{kV}$, a gap distance of $17 \mathrm{~cm}$ and a feed rate of $30 \mu \mathrm{l} / \mathrm{min}$. Three additional ring electrodes with a decreasing potential along the distance from the needle tip, and with a separation of $2 \mathrm{~cm}$, were used to homogenize the primary electric field and to extend the stable region of the jet. Due to the applied ring potentials, the resulting constant component of the electric field strength was $2.21 \mathrm{kV} / \mathrm{cm}$ for the first $4 \mathrm{~cm}$ (zone 1 ) and $1.36 \mathrm{kV} / \mathrm{cm}$ from there to the collector plate (zone 2). These values were calculated from the applied electrode potentials and the electrode separation and represent average field strengths within the described zones. Two deflector plates were mounted perpendicular to the jet direction and connected to a high voltage amplifier (TReK 610E) facilitating vertical alternating electric fields by amplifying signals generated by a National Instruments NI-USB 6211. The lower constant electric field strength in zone 2 was chosen in order to maximise the effect of the superimposed dynamic field generated by the deflector plates (max. $\pm 10 \mathrm{kV}$ ). 210 membranes were spun, investigating different field signal types (square, triangle, sine), deflector plate amplitudes $\left(U_{d}=2 \pm 6 k V, 2 \pm 8 k V\right.$, which results in peak to peak voltages of $\left.V_{p p}=12 \mathrm{kV}, 16 \mathrm{kV}\right)$ and deflection frequency ranges $\left(f_{d}=1 . .160 \mathrm{~Hz}, 0.5 . .10\right.$ $\mathrm{Hz}$ ) as shown in Table 1. The spun membranes were removed from the collector plate and degassed for several days in a desiccator before further analysis.

\subsection{Sample preparation for SEM imaging and mechanical testing}


For the fibre alignment experiment, small discs with a diameter of $6 \mathrm{~mm}$ were punched out of the membranes at the regions of interest. Four different locations were investigated: top side centre (tc), side (ts) and top (tt) region as well as bottom side centre (bc) region (Figure 2a). For identification of the deflection axis within the scanning electron microscope, an additional cut was made at the edge of the samples. For mechanical testing, $12 \mathrm{~mm}$ wide rectangular strips were cut from a subset of the membranes either in the direction of the deflection axis or perpendicular to it (Figure 2a). For these samples, the punch for SEM imaging was taken right next to the cut strip, again including an edge of the cut in order to identify the test direction within the SEM image. After gold sputter coating, SEM images were acquired with an FEI Quanta 200 FEG, using the back-scattered electron detector (BSED). Since fibre diameter and orientation, but not the fibre surface, were of interest in this study, contrast was set high in order to achieve bright white fibres standing out from the very dark background of lower fibre layers and pore spaces (Figure $2 b$ )

\subsection{Fibre diameter and orientation analysis}

12 SEM images taken at different positions on each punch were analysed using ImageJ (1.49k, Wayne Rasband, National Institutes of Health, USA). In a first step, fibre diameter distribution and mean fibre diameter were calculated based on a method described by Ziabari et al. (2007). In short, after a threshold operation, a distance map, as well as a skeleton of the image or fibre mesh, is calculated. Multiplication of both these images results in a skeleton, where each pixel value of the skeleton represents the radius of the fibre at that particular position. After removal of fibre crossings, as 
there the calculated distance is higher than the actual fibre diameter, the histogram of this image counting all values greater than zero represents the fibre diameter distribution in pixels. Fibre orientation analysis was performed using the OrientationJ (Rezakhaniha et al., 2011) plugin for ImageJ, resulting in an orientation distribution histogram for each image. OrientationJ uses a pixel-wise algorithm that calculates a 2D structure tensor, resulting in an orientation, energy and coherency map. The orientation of each pixel corresponds to the direction of the largest eigenvector of the tensor and is weighted by its coherency, which is defined as the ratio between the difference and the sum of the tensor eigenvalues (Rezakhaniha et al., 2011). Based on this data, a fibre orientation histogram was calculated and normalized to the total amount of pixels $(\widehat{H}(\theta))$, while the average fibre radius was used as the window size for the structure tensor calculation. Figure $2 c$ shows a colour coded output of the analysed SEM image of Figure $2 \mathrm{~b}$. The main fibre direction $(\Delta \theta)$ in relation to the deflection direction, as well as variance of fibre orientation within the image, was calculated for each acquired image, while $\theta=0$ deg corresponds to the horizontal direction in each image (Figure 2d). For the calculation of variance in fibre orientation, the main fibre direction was rotated by rot into the centre of the histogram. The variance measures how far the fibre orientations spread around the main fibre direction, hence is a measure of fibre alignment.

$$
\begin{gathered}
\operatorname{Var}\left(\widehat{H}\left(\theta^{\prime}\right)\right)=\frac{1}{n} \sum_{i=1}^{n}\left(\theta^{\prime}{ }_{i}-{\overline{\theta^{\prime}}}^{2} \quad\left[\text { deg }^{2}\right]\right. \\
\text { with } \quad \theta^{\prime}=\theta+\text { rot }
\end{gathered}
$$


The orientation of the deflection axis or tensile test axis $(\varphi)$, respectively, was measured manually using ImageJ by taking 3 measurements for each punch, resulting in a standard deviation of $<0.5$ degrees correspondingly (Figure $2 b$, inlay).

\subsection{Mechanical testing and analysis}

The previously described $12 \times 60 \mathrm{~mm}$ strips cut from a subset of the spun scaffolds were tested for their tensile properties using an Instron ElectroPuls E10000, after membrane thickness was measured using a thickness gauge (Käfer Messuhren, Villingen-Schwenningen, Germany) at a standardised contact pressure of $7.85 \mathrm{kPa}(1 \mathrm{~N}$, $\varnothing 10 \mathrm{~mm}$ ) and with an accuracy of $0.01 \mathrm{~mm}$. The cross-section area was calculated from the membrane width $(\mathrm{w}=12 \mathrm{~mm})$ and the measured membrane thickness. The membranes were stretched at a rate of $0.5 \mathrm{~mm} / \mathrm{s}$ until failure or to a maximum strain of $285 \%$ (maximum stroke of the testing device), respectively. Stress and strain were calculated from the measured force and displacement values. Young's modulus was calculated using a linear fit to the linear elastic part of the stress-strain curve with $R^{2}>$ 0.99. Yield stress was defined from the intersection of a $2 \%$ offset of the linear elastic fit with the stress-strain curve (Figure $2 f)$. The relevant fibre ratio parallel $\left(P_{\Perp}\right)$ to the test direction was calculated for each image by summing up the parallel fractions of the fibre orientation histogram (Figure 2e), whereas $\widehat{H}(\alpha)$ represents the orientation distribution histogram normalised to the total amount of pixels. $\alpha=0$ deg corresponds to the testing direction and can be calculated by a rotation of $\theta: \alpha=\theta-\varphi$.

$$
\widehat{H}(\alpha) * \cos (\alpha)=\widehat{H}_{\mathrm{II}}(\alpha)
$$




$$
P_{I I}=\sum^{\alpha} \widehat{H}_{I I}(\alpha)
$$

\subsection{Statistical analysis}

For fibre alignment, Levene's tests were conducted to test for a statistically significant difference between each of the groups. The Levene's test assesses the assumption that the variances of populations from which the samples are drawn, and hence the degrees of fibre alignment, are equal. This method of analysis was chosen, since Shapiro-Wilk tests of the fibre orientation histograms revealed that these do not exhibit a normal distribution ( $p<0.001)$, which is a requirement for the most common analysis methods. To test for statistical significance of the main fibre direction compared to the deflection axis, a Student's t-test has been performed, while an analysis of variance has been used to assess the influence of fibre alignment on the fibre diameter and the tensile properties.

\section{Results}

\subsection{Fibre diameter and main fibre direction}

Mean fibre diameter showed a modest but significant $(p<0.01)$ increase from 1.92 to $2.07 \mu \mathrm{m}$ (Figure 3) with increasing fibre alignment. There was no statistically significant influence of the degree of fibre alignment on the main fibre direction relative to the deflection axis ( $\Delta \theta$, Figure 4). However, contrary to the initial hypothesis, the main fibre direction proved to be not in line with the deflection axis, but rather perpendicular to it. The experiment revealed a mean $\Delta \theta=87 \pm 18$ deg with a $95 \%$ confidence interval of [83.92 ... 90.08] deg. 


\subsection{Fibre alignment}

In a first round, frequencies $\mathrm{fd}=[10,20,40,80,120,160] \mathrm{Hz}$ at a deflector voltage amplitude $V_{p p}=12 \mathrm{kV}$ have been investigated, since as an initial guess, fibre alignment was expected to occur between 20 and $80 \mathrm{~Hz}$. This guess was based on a simplified calculation of the fibre time of flight, considering feed rate, final mean fibre diameter (gained from a previous experiment with a similar setup) and hence an estimated fibre deposition rate. Fibre orientation analysis revealed that there were significant differences between the investigated deflection frequencies. The best fibre alignment was found to be in the low frequency range at 10 and $20 \mathrm{~Hz}$, while alignment decreased with increasing frequency. Due to this observation, in a second round the frequencies $f_{d}=[0.5,1,2,4,7,10] \mathrm{Hz}$ were investigated. The influence of deflector voltage amplitude has been investigated by spinning at $V_{p p}=12 \mathrm{kV}$ and $V_{p p}=16 \mathrm{kV}$. The higher deflector voltage amplitude lead to a better fibre alignment, however $\mathrm{V}_{\mathrm{pp}}=16 \mathrm{kV}$ represented the maximum applicable amplitude without fibres being deposited also on the deflector plates. The parameter sets resulting in the best alignment were $f_{d}=[2,10] \mathrm{Hz}$ at $V_{p p}=16 \mathrm{kV}$, expressing an average variance in fibre orientation of $144 \mathrm{deg}^{2}$ and $149 \mathrm{deg}^{2}$ respectively (Figure 5). Furthermore, while the 16 $\mathrm{kV}$ group has its minimum in variance between 2 and $10 \mathrm{~Hz}$, the $12 \mathrm{kV}$ group shows a similar minimum between 1 and $4 \mathrm{~Hz}$ with variances of $317 \mathrm{deg}^{2}$ and $329 \mathrm{deg}^{2}$ respectively. Concerning the regions of interest, as described above, there is a significant difference in fibre alignment depending on the region of the spun membrane. The first layers of fibres that are deposited (the bottom side of the membrane) are laid down in very small loops, hence resulting in a random fibre 
alignment in the orientation analysis. The top side of the membrane consists of much larger loops, appearing as straight fibres within the SEM images, which can either be aligned or show a random distribution of orientation, depending on location on the membrane. However, bottom side (bc) samples always feature these small fibre loops resulting in a random fibre orientation, while the best alignment could be observed for top side samples in the centre region (tc). The top side and top top regions of the membranes (ts and $\mathrm{tt}$ ) exhibit as expected less aligned fibres compared to the centre region (Figure 6). Figure 7 shows 2 aligned/random aligned sample images to give an impression of the degree of alignment that could be achieved. For the parameter set with the overall best alignment (triangle wave, $2 \mathrm{~Hz}, 16 \mathrm{kV}$ deflection) on average $61 \pm 10 \%$ of all fibres were within \pm 10 degrees, while $87 \pm 9 \%$ of all fibres were within \pm 20 degrees of main fibre orientation. The differences in alignment due to the different deflection signals (square, triangle and sine) are small compared to the effect of the frequency changes. A comparison between the signal types is provided as supplementary data.

\subsection{Tensile properties}

The tested membrane strips had an average thickness of $0.53 \pm 0.10 \mathrm{~mm}$. As expected, fibre alignment, or rather membrane anisotropy, is reflected in its tensile properties. Young's modulus and yield stress increased with the ratio of the parallel fibre component from min. 2.0 to max. 9.3 $\mathrm{MPa}$ and 0.1 to $0.7 \mathrm{MPa}$ respectively (Figure 8). Furthermore a direct correlation between Young's modulus and yield stress was observed, while all correlations drawn are highly significant with $p<0.001$. No 
conclusive result, however, can be drawn concerning ultimate tensile stress and failure strain, since $28 \%$ of all tested samples did not fail before the maximum travel of the testing device was reached, which corresponds to a strain of $285 \%$. Nevertheless, minimum failure strain and minimum ultimate tensile stress was observed at $60.0 \%$ and $0.48 \mathrm{MPa}$ respectively.

\section{Discussion}

The use of a dynamic, alternating electrical field perpendicular to the electrospinning direction, as a means to directly control fibre alignment, has been evaluated. The feasibility of the described method to achieve fibre alignment could be demonstrated. Low frequency deflection seems to give the natural whipping effect of the polymer jet a preferred direction and leads to better alignment than direct high frequency fibre deposition attempts. However, contrary to the initial hypothesis, the main fibre direction is not aligned with the deflection axis but consistently perpendicular to it, which is also reflected in the tensile properties of spun samples. This result is surprising, as Grasl et al. (2013) reported fibre alignment in the deflection direction when spinning PEO and applying a high frequency $(30 \mathrm{~Hz}-60 \mathrm{~Hz})$ square wave deflection signal - an effect which was not observed in the present study. However, the ratio of the dynamic field strength generated by the deflector plates and the static field driving the spinning process in the setup used by Grasl et al. was 2-3 times higher than that used in the present study. This higher deflection gradient also resulted in fibre deposition on the deflector plates in the low frequency range. The observed orientation of the main fibre direction perpendicular to the deflection axis in the low 
frequency range may be due to the compression effect described by Nurfaizey et al. (2011). As the whipping frequency of the jet is very high, the low deflection frequencies are likely to steer the entire instability to oscillate parallel to electrode axis, similar to what was observed by Grasl et al. (2013) when spinning in "focus mode". However, if the instability is compressed then it is likely to result in the deposited loops being elongated perpendicular to the electrode axis giving the appearance of alignment, which also accounts for the higher degree of orientation observed in the centre of the membrane compared to the side regions. The spatial variation in alignment observed in the present study, and the dependence of fibre deposition width on deflection field potential reported by Grasl et al (2013) - not observed in the present study - represent two challenges to the final application of such methods.

Grasl et al. reported a $40 \%$ increase in fibre diameter between the tested deflection modes. In the present study, only small changes could be observed in fibre diameter for all tested parameter sets, while also applying a constant feed rate. The superimposed dynamic electric field seems to have a minor effect on the fibre deposition rate. Furthermore, due to the auxiliary ring electrodes, which are focusing the electric field within the first $4 \mathrm{~cm}$ of the electrospinning setup, jet deflection had no influence on jet initiation. Nevertheless fibre diameter measurements are based on a graphical analysis of SEM images, without verification by e.g. optical microscopy, and hence are sensitive to the magnification, or rather the physical pixel size, which represents the maximum resolution of the measurement - an observation that has just recently been confirmed by Stanger et al. (2014). In fact, fibre diameter analysis from 
images with doubled magnification but from the same region resulted in a $10 \%$ drop in average fibre diameter, when choosing rather low magnifications, where fibre diameters are of just a few pixels. For this reason, all fibre diameter measurements and their statistical comparison were carried out on images with the same physical pixel size in order to ensure inter-and intra-membrane comparability.

Bottom up samples, representing the initially deposited layer of the electrospun membrane, feature very small diameter fibre loops, leading to a random fibre orientation in the analysis. These loops get bigger with the amount of charge trapped in the polymer or membrane and hence accumulating on the collector. This residual potential within the membrane likely has a substantial influence on the electric field, whereas the decay is quite slow compared the time of the electrospinning process. Collins et al. (2012) describes an initial residual potential of more the $1 \mathrm{kV}$ with a halflife $>10$ hours in electrospun polystyrene membranes and also Ignatova et al. (2008) observed a rather strong decay of the surface potential within a time frame of 10 days for spun PET membranes, while an elevated potential was evident for up to 50 days. However this effect seems to be advantageous for fibre alignment using deflector plates, since the jet instability in the initial spinning phase with a "clean" collector might not be strong enough to be influenced by the alternating deflection field, whereas this might be the case with increasing jet instability. This observation agrees with the fact that fibre alignment could not be achieved by high frequency deflection, but by influencing the inherent bending instability in the low frequency range. Nonetheless, strong fibre alignment can be observed on the top side images for triangle and sine wave deflection signals between 2 and $10 \mathrm{~Hz}$. However, the change in 
alignment from the bottom to the top side has not been specifically investigated in this study. It is not clear whether there is a gradient throughout the sample or a narrow transition zone followed by a rather constant degree of alignment.

As there is no consistent definition of fibre alignment, comparisons to studies conducted by other groups are rather difficult. Milleret et al. (2011) measured fibre orientation manually with ImageJ and defined all fibres with a maximum angular deviation of \pm 10 degrees from the mean orientation as aligned. Using the rotating mandrel method, Milleret et al. were able to produce perfectly aligned fibre mats with an alignment of $100 \%$ (1330 rpm, mandrel diameter: $80 \mathrm{~mm}$ ), by their definition. Calculating this parameter from the orientation histograms of the present study, the maximum alignment that could be achieved in a single membrane was at $91.2 \%$ (triangle, $f_{d}=10 \mathrm{~Hz}, V_{p p}=16 \mathrm{kV}$ ), while the best average alignment was found for sine deflection at $1 \mathrm{~Hz}$ with 68.2\%. Li et al. (2007) defined \pm 20 degrees as a threshold for aligned fibres and were able to reach a maximum alignment of $94 \%$ with the fastest mandrel rotation also spinning PCL. Setting that as threshold for the present study an average alignment of 92,6\% again for the $1 \mathrm{~Hz}$ sine deflection group could be achieved. Gaumer et al. (2009) also used a pixel-wise algorithm based on a watershed transformation and calculated the standard deviation from the mean orientation of PCL fibres spun onto a mandrel with up to $10 \mathrm{~m} / \mathrm{s}$ surface velocity. They observed standard deviations down to $30 \mathrm{deg}$, which equals a variance of $900 \mathrm{deg}^{2}$, while with the tested jet deflection method of the present study a minimum variance of $140 \mathrm{deg}^{2}$ could be achieved. In fact, all sample groups, which were spun with a deflection frequency $<20 \mathrm{~Hz}$ show a variance in fibre orientation of less than $900 \mathrm{deg}^{2}$. 
Therefore, dynamic jet deflection leads to similar alignment results as the methods based on fast collector movement, however, a perfect alignment, as presented by Milleret et al., could not be achieved. But what degree of fibre alignment is desired for tissue engineering applications? Highly aligned fibres also lead to a decrease in pore size, which is disadvantageous for cell infiltration. On the other hand fibre alignment can also guide cells, influence their fate, differentiation, behaviour and morphology (Ionescu and Mauck, 2013). In many cases, a densely packed, highly aligned structure is not desirable for tissue engineering applications.

Inter-sample variability was observed to be very high. This might be due to the fact that the laboratory spinning setup did not allow to explicitly control the atmospheric environment such as temperature, pressure and most importantly humidity. Although quite constant (temperature $22^{\circ} \mathrm{C}-25^{\circ} \mathrm{C}$, humidity $<45 \%$ ), variation in these factors can lead to differences in the spinning result (Rnjak-Kovacina and Weiss, 2011; Theron et al., 2004). Nevertheless, due to the large amount of samples, significant results could still be acquired.

Grasl et al. (2013) previously demonstrated the potential for fibre alignment with high frequency dynamic field switching, but did not report the influence of the thusachieved alignment on membrane properties. As shown in Figure 8 , the tensile properties reflect nicely the observed fibre alignment. Young's modulus (2.0 - 9.3 MPa) as well as yield $(0.1-0.7 \mathrm{MPa})$ increased with the fraction of fibres parallel to the test direction. However, the spun membranes and hence the test strips did not present a perfect uniform thickness, as would be the case for mandrel spun membranes. Nonetheless, the acquired mechanical properties agree with measurements by Li et al. 
(2007), who observed a young's modulus of $2.1 \pm 0.4 \mathrm{MPa}$ for random aligned PCL membranes and $11.6 \pm 3.1 \mathrm{MPa}$ for aligned membranes tested in fibre direction. However, these membrane strips possessed a smaller fibre diameter, which tends to exhibit also lower Young's modulus and yield strength, compared to thicker fibre diameter membranes. Gaumer et al. report significantly higher moduli and yield strength of $5-38 \mathrm{MPa}$ and $2-10 \mathrm{MPa}$ respectively for PCL membranes, depending on fibre alignment, but do not state an average fibre diameter. However, the presented SEM images and the higher concentration polymer spinning solution indicate a larger fibre diameter compared to those achieved in the present study. Nevertheless, the reported increase in tensile modulus and yield strength for aligned compared to random aligned fibres $(250-300 \%)$ is similar to that found in the present study. Baker et al. (2008) also report tensile moduli from 1.4 to $16.1 \mathrm{MPa}$ for pure PCL membranes parallel and perpendicular to fibre direction, while again fibre diameter has not been reported. A direct correlation was observed between Young's modulus and yield stress, an effect also observable in natural soft tissues. With a highly aligned membrane, most fibres respond simultaneously, presenting a quite stiff behaviour (high modulus) even upon initial loading, and reaching their ultimate failure strain together, resulting in a high yield stress as well. For a randomly aligned membrane however, there is a gradual recruitment of fibres and progressive alignment, hence a lower modulus. At higher strains, individual fibres initially aligned with the loading direction fail first, followed by a progressive failure of fibres throughout the membrane, hence a lower effective yield stress is observed. 


\section{Conclusion}

In conclusion, it is possible to achieve fibre alignment directly by dynamic jet deflection, independent of collector geometry or motion (and their limits). The achieved alignment is comparable to other methods, also in relation to the change in mechanical properties. As shown above, with the described electrospinning setup and parameters, a ratio of aligned fibres ( $\pm 20 \mathrm{deg}$ ) of $87 \pm 10 \%$ for $2 \mathrm{~Hz}$, triangle wave deflection can be achieved. However the alignment decreases towards the side regions of the spun membranes, which constitutes a limitation of this technique, or at least a consideration when selecting regions of the spun membranes for post-processing. Nevertheless, depending on the desired scaffold shape and application, dynamic jet deflection can be a feasible alternative to the moving collector methods.

\section{Acknowledgements}

Technical assistance of Jonas Widmer is gratefully acknowledged. This study is part of the NCCR Co-Me project "Cartilage Tissue Engineering for ORL Applications", the AO Collaborative Research Program for annulus fibrosus repair, and was funded by the Swiss National Science Foundation and the AO Foundation. The authors acknowledge support by the Scientific Center for Optical and Electron Microscopy of ETH Zurich (SCOPEM). 


\section{References}

Arras MML, Grasl C, Bergmeister H, Schima H (2012) Electrospinning of aligned fibers with adjustable orientation using auxiliary electrodes. Sci. Technol. Adv. Mater. 13: 035008. doi:10.1088/1468-6996/13/3/035008.

Baji A, Mai Y-W, Wong S-C, Abtahi M, Chen P (2010) Electrospinning of polymer nanofibers: Effects on oriented morphology, structures and tensile properties. Compos. Sci. Technol. 70: 703-718. doi:10.1016/j.compscitech.2010.01.010.

Baker BM, Gee AO, Metter RB, Nathan AS, Marklein R a, Burdick J a, Mauck RL (2008) The potential to improve cell infiltration in composite fiber-aligned electrospun scaffolds by the selective removal of sacrificial fibers. Biomaterials 29: 2348-58. doi:10.1016/j.biomaterials.2008.01.032.

Bhardwaj N, Kundu SC (2010) Electrospinning: a fascinating fiber fabrication technique. Biotechnol. Adv. 28: 325-47. doi:10.1016/j.biotechadv.2010.01.004.

Collins G, Federici J, Imura Y, Catalani LH (2012) Charge generation, charge transport, and residual charge in the electrospinning of polymers: A review of issues and complications. J. Appl. Phys. 111: 044701. doi:10.1063/1.3682464.

Courtney T, Sacks MS, Stankus J, Guan J, Wagner WR (2006) Design and analysis of tissue engineering scaffolds that mimic soft tissue mechanical anisotropy. Biomaterials 27: 3631-8. doi:10.1016/j.biomaterials.2006.02.024.

Gaumer J, Prasad A, Lee D, Lannutti J (2009) Structure-function relationships and source-to-ground distance in electrospun polycaprolactone. Acta Biomater. 5: 155261. doi:10.1016/j.actbio.2009.01.021.

George JHS (2011) Engineering of Fibrous Scaffolds for use in Regenerative Medicine; Imperial College London.

Grasl C, Arras MML, Stoiber M, Bergmeister H, Schima H (2013) Electrodynamic control of the nanofiber alignment during electrospinning. Appl. Phys. Lett. 102: 7-11. doi:10.1063/1.4790632.

Heikkilä P, Söderlund L, Uusimäki J, Kettunen L, Harlin A (2007) Exploitation of electric field in controlling of nanofiber spinning process. Polym. Eng. Sci. 47: 2065-2074. doi:10.1002/pen.20923.

Ignatova M, Yovcheva T, Viraneva A, Mekishev G, Manolova N, Rashkov I (2008) Study of charge storage in the nanofibrous poly(ethylene terephthalate) electrets prepared by electrospinning or by corona discharge method. Eur. Polym. J. 44: 1962-1967. doi:10.1016/j.eurpolymj.2008.04.027. 
Ionescu LC, Mauck RL (2013) Porosity and cell preseeding influence electrospun scaffold maturation and meniscus integration in vitro. Tissue Eng. Part A 19: 538-47. doi:10.1089/ten.TEA.2012.0052.

Kim GH (2008) Electrospun PCL nanofibers with anisotropic mechanical properties as a biomedical scaffold. Biomed. Mater. 3: 025010. doi:10.1088/1748-6041/3/2/025010.

Kumbar SG, James R, Nukavarapu SP, Laurencin CT (2008) Electrospun nanofiber scaffolds: engineering soft tissues. Biomed. Mater. 3: 034002. doi:10.1088/1748$6041 / 3 / 3 / 034002$.

Li W-J, Mauck RL, Cooper J a, Yuan X, Tuan RS (2007) Engineering controllable anisotropy in electrospun biodegradable nanofibrous scaffolds for musculoskeletal tissue engineering. J. Biomech. 40: 1686-93. doi:10.1016/j.jbiomech.2006.09.004.

Milleret V, Simona B, Neuenschwander P, Hall H (2011) Tuning electrospinning parameters for production of 3D-fiber-fleeces with increased porosity for soft tissue engineering applications. Eur. Cell. Mater. 21: 286-303.

Nerurkar NL, Elliott DM, Mauck RL (2007) Mechanics of oriented electrospun nanofibrous scaffolds for annulus fibrosus tissue engineering. J. Orthop. Res. 25: 101828. doi:10.1002/jor.20384.

Nisbet DR, Forsythe JS, Shen W, Finkelstein DI, Horne MK (2009) Review paper: a review of the cellular response on electrospun nanofibers for tissue engineering. J. Biomater. Appl. 24: 7-29. doi:10.1177/0885328208099086.

Nurfaizey AH, Stanger J, Tucker N, Buunk N, Wallace A, Staiger MP (2011) Manipulation of electrospun fibres in flight: the principle of superposition of electric fields as a control method. J. Mater. Sci. 47: 1156-1163. doi:10.1007/s10853-011-5847-3.

Nurfaizey AH, Stanger J, Tucker N, Buunk N, Wood AR, Staiger MP (2014) Control of Spatial Deposition of Electrospun Fiber Using Electric Field Manipulation. J. Eng. Fabr. Fibers 9: 155-164.

Pham QP, Sharma U, Mikos AG (2006) Electrospinning of polymeric nanofibers for tissue engineering applications: a review. Tissue Eng. 12: 1197-211. doi:10.1089/ten.2006.12.1197.

Rezakhaniha R, Agianniotis A, Schrauwen JTC, Griffa A, Sage D, Bouten CVC, van de Vosse FN, Unser M, Stergiopulos N (2011) Experimental investigation of collagen waviness and orientation in the arterial adventitia using confocal laser scanning microscopy. Biomech. Model. Mechanobiol. 11: 461-73. doi:10.1007/s10237-0110325-z. 
Rnjak-Kovacina J, Weiss AS (2011) Increasing the pore size of electrospun scaffolds. Tissue Eng. Part B. Rev. 17: 365-72. doi:10.1089/ten.teb.2011.0235.

Secasanu VP, Giardina CK, Wang Y (2009) A novel electrospinning target to improve the yield of uniaxially aligned fibers. Biotechnol. Prog. 25: 1169-75.

doi:10.1002/btpr.163.

Stanger JJ, Tucker N, Buunk N, Truong YB (2014) Test method A comparison of automated and manual techniques for measurement of electrospun fi bre diameter. Polym. Test. 40: 4-12. doi:10.1016/j.polymertesting.2014.08.002.

Teo WE, Ramakrishna S (2005) Electrospun fibre bundle made of aligned nanofibres over two fixed points. Nanotechnology 16: 1878-1884. doi:10.1088/0957$4484 / 16 / 9 / 077$.

Theron S a., Zussman E, Yarin a. L (2004) Experimental investigation of the governing parameters in the electrospinning of polymer solutions. Polymer (Guildf). 45: 20172030. doi:10.1016/j.polymer.2004.01.024.

Ziabari M, Mottaghitalab V, McGovern ST, Haghi a. K (2007) A New Image Analysis Based Method for Measuring Electrospun Nanofiber Diameter. Nanoscale Res. Lett. 2: 597-600. doi:10.1007/s11671-007-9093-1. 
Figure 1: Electrospinning setup. Fixed parameters: $\mathrm{U}_{0}=26.5 \mathrm{kV}, \mathrm{U}_{1}=22.1 \mathrm{kV}, \mathrm{U}_{2}=$ $17.7 \mathrm{kV}, \mathrm{d}_{\text {gap }}=17 \mathrm{~cm}, \mathrm{~d}_{\mathrm{aux}}=2 \mathrm{~cm}, \mathrm{R}_{1}=7 \mathrm{G} \Omega, \mathrm{I}_{\text {defl }}=7 \mathrm{~cm}, \mathrm{~d}_{\text {defl }}=12 \mathrm{~cm}, \mathrm{v}_{0}=30 \mu \mathrm{l} / \mathrm{min}$, $U_{d}, f_{d}$ : varying. $U_{d}=2 \pm 6 \mathrm{kV}$ corresponds to $V_{p p}=12 \mathrm{kV}$ (peak-peak), while $U_{d}=2 \pm 8 \mathrm{kV}$ corresponds to $\mathrm{V}_{\mathrm{pp}}=16 \mathrm{kV}$.

Figure 2: Methods. (a) Punch positions for alignment (left) and mechanical tests (right). (b) Example SEM image (BSED) for orientation analysis. The small inlay shows the determination of the reference axis (deflection and/or test direction). (c) Same SEM image after analysis, fibre orientation colour coded (HUE). (d) Fibre orientation histogram with marked main directions and parameter definitions: in grey the original histogram calculated from the image $(\hat{H}(\theta))$, in blue the rotated one $(\hat{H}(\theta+r o t))$ for the analysis of variance in fibre orientation. (e) Exemplary fibre orientation histogram illustrating the calculation of the parallel fibre ratio $\left(P_{\|}\right)$, which is represented by the (blue) area under the $H_{\|}(\alpha)$ curve. (f) Stress-strain curve of a tested membrane stripe (blue). The red curve represents the average stress-strain curve of all tested samples spun with the same parameter set (here: sine, $1 \mathrm{~Hz}, \mathrm{~V}_{\mathrm{pp}}=16 \mathrm{kV}$ ), while its standard deviation is plotted in grey. Young's modulus was calculated using a linear fit (green dashed line) to the linear elastic part of the stress-strain curve, while yield stress (grey horizontal line) was defined from the intersection of a $2 \%$ offset of the linear elastic fit with the stress-strain curve.

Figure 3: Main fibre diameter. Colour and symbol coded scatterplot of the measured main fibre diameter compared to the fibre alignment and deflection signal waveform. Fibre alignment has a modest but significant influence $(p<0.01)$ on mean fibre diameter, which increases with fibre alignment from 1.92 to $2.07 \mu \mathrm{m}$, while the 
triangle wave deflection signal yielded a significantly $(p<0.001)$ lower fibre diameter compared to the sine wave deflection signal.

Figure 4: Main fibre direction. Dominant fibre direction compared to the deflection axis and variance in fibre orientation. Fibres consistently align perpendicular to the deflection axis, independently from fibre alignment; $\Delta \theta=87 \pm 18 \mathrm{deg}$.

Figure 5: Fibre alignment vs. deflection voltage (peak-peak; $\left.V_{p p}\right)$ and frequency $\left(f_{d}\right)$. All included measurements were taken from the tc region of the spun scaffolds. Pairs marked with (\#) showed no significant difference. All other pairwise comparisons between the groups showed a significant difference with $p<0.05$ (not marked in the figure).

Figure 6: Fibre alignment vs. punch position (scaffold region). Regions: (tt) top side up - top, (ts) top side up - side, (tc) top side up-centre, (bc) bottom side up - centre. All differences within the 4 plotted regions are significant $(p<0.05)$.

Figure 7: Example of aligned vs. random aligned fibres. (left) original images; (right) colour coded analysed images (Hue: orientation, saturation: coherency, brightness: energy); (top) sample spun with a $2 \mathrm{~Hz}$ triangle wave deflection signal; (bottom) sample spun with a $80 \mathrm{~Hz}$ triangle wave deflection signal; (centre) fibre orientation histogram for both images.

Figure 8: Tensile properties compared to fibre alignment. Young's modulus and yield stress plotted versus the parallel fibre ratio and each other. All drawn linear correlations are significant with $p<0.001$. The horizontal bars in the left and centre plot indicate the standard deviation of parallel fibre ratios, which were calculated from different spots of the same sample. 


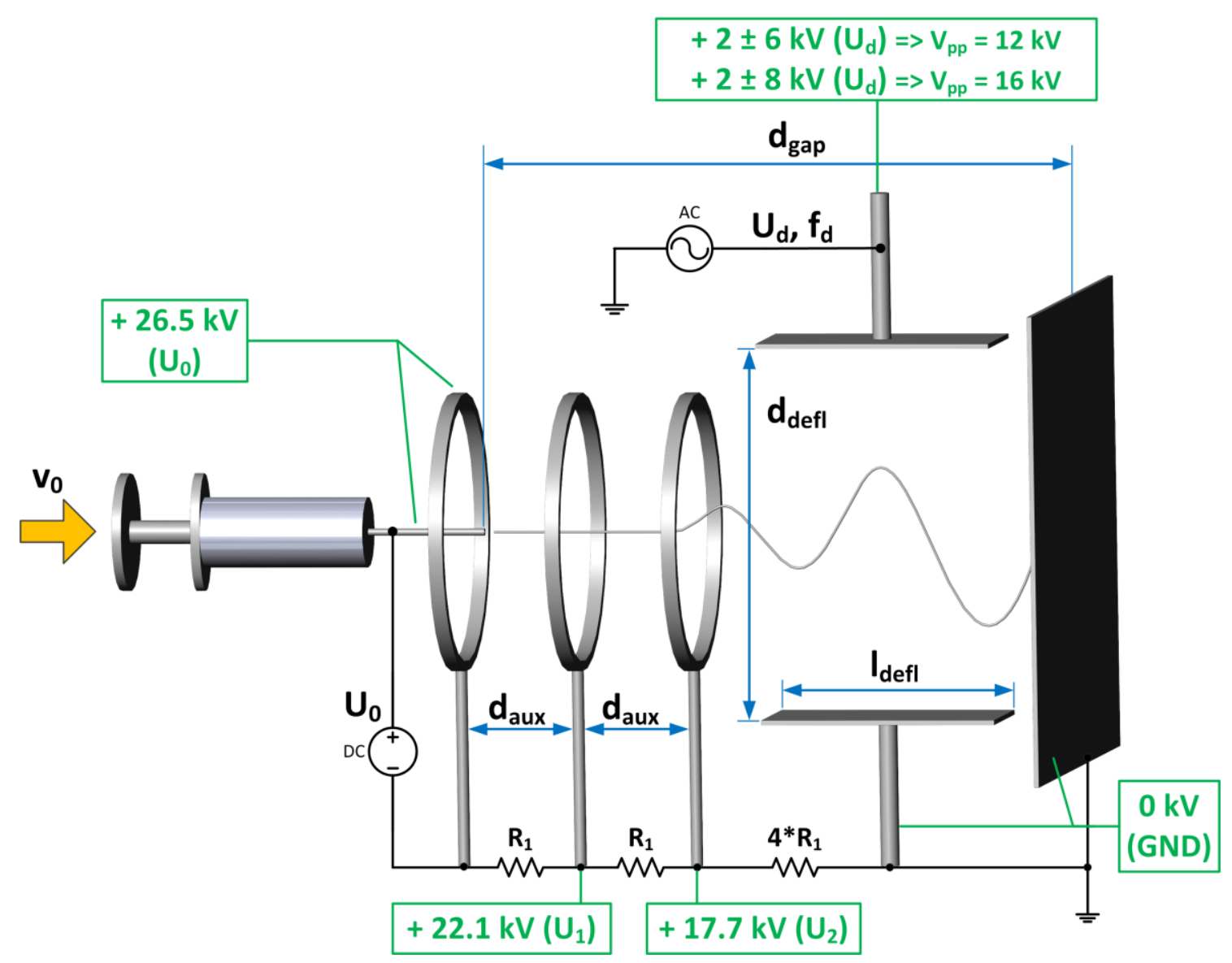




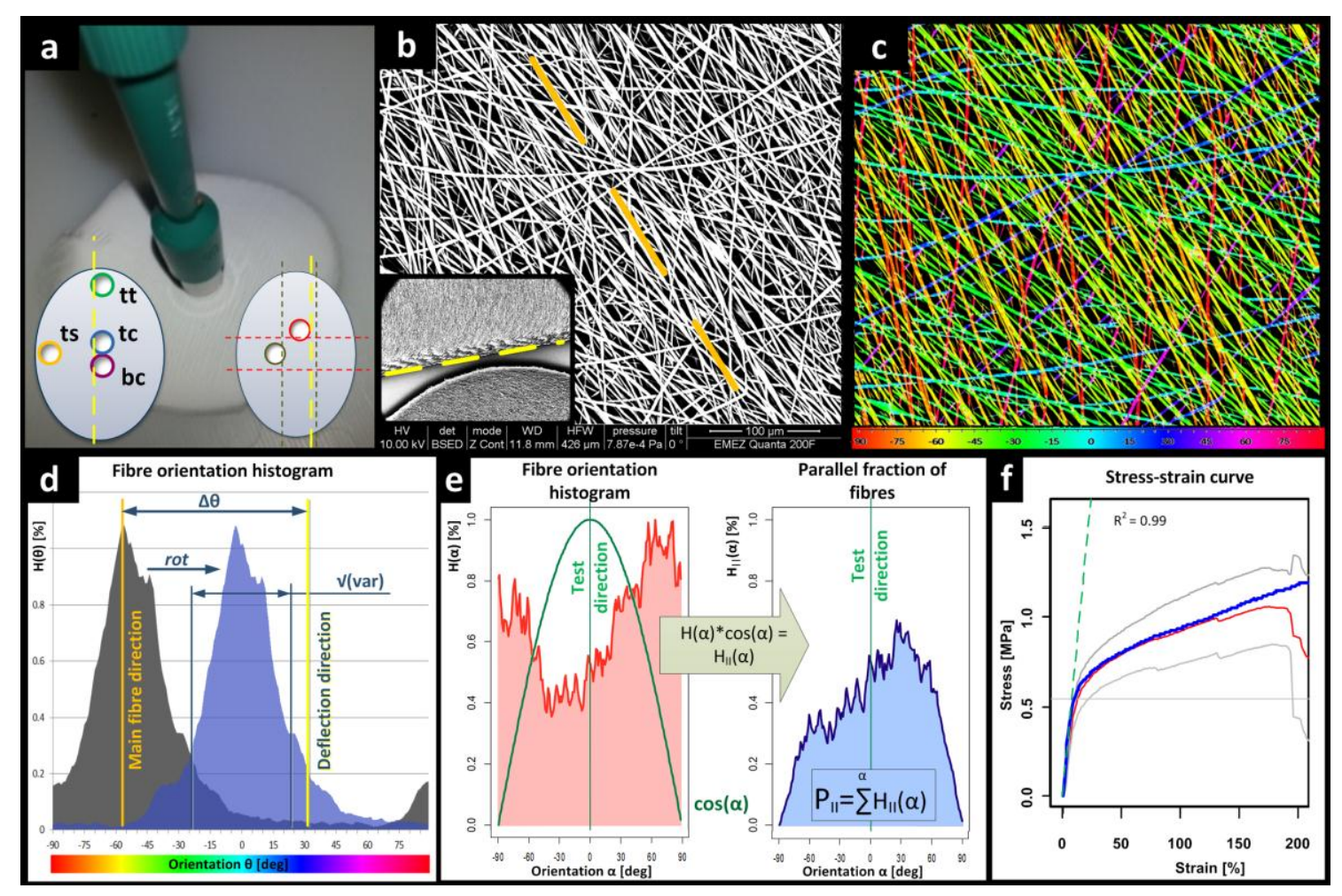


Mean fibre diameter vs. fibre alignment and deflection signal
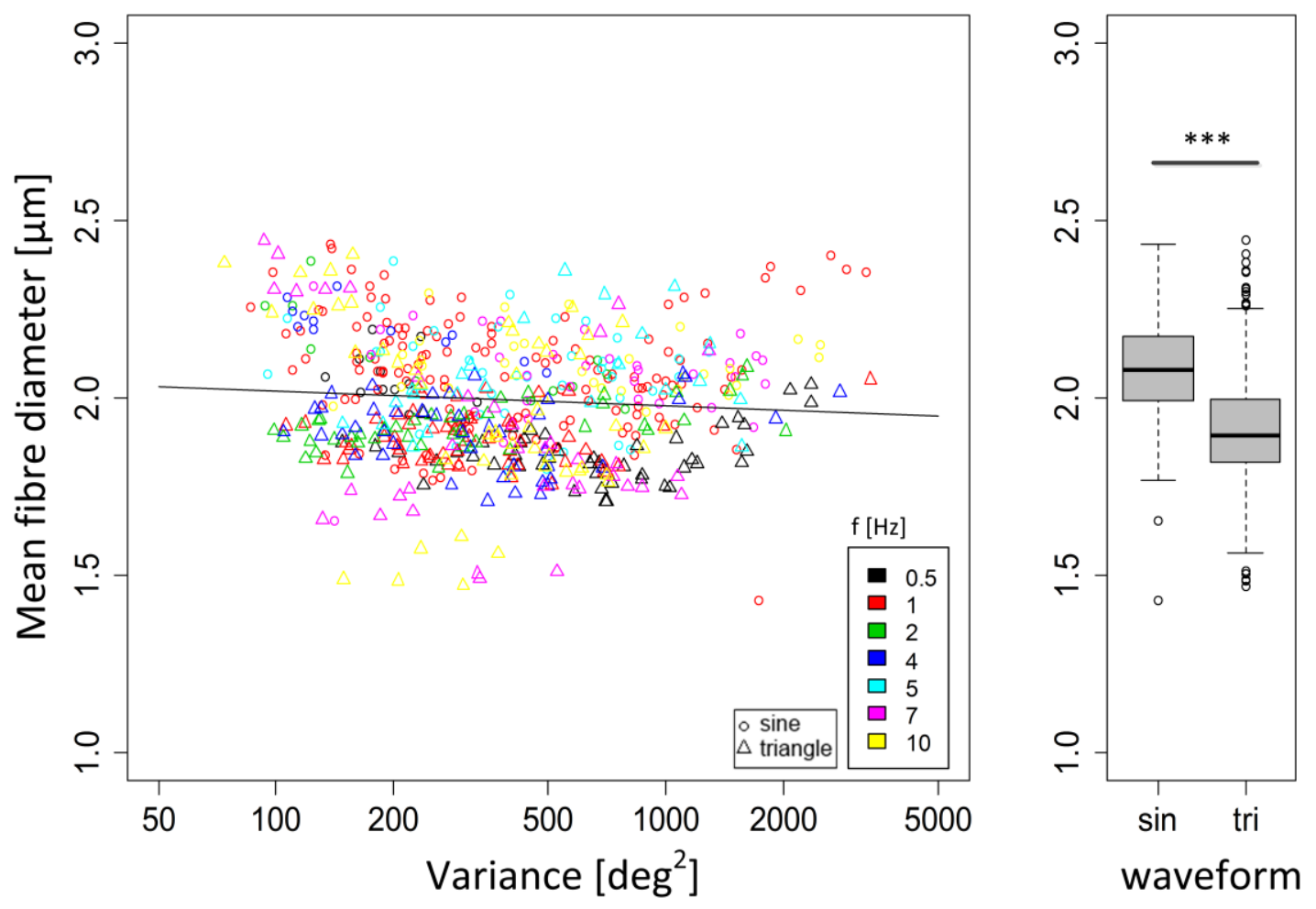
Main fibre direction in relation to deflection axis $(\Delta \alpha)$ vs. Fibre alignment and frequency (centre region)
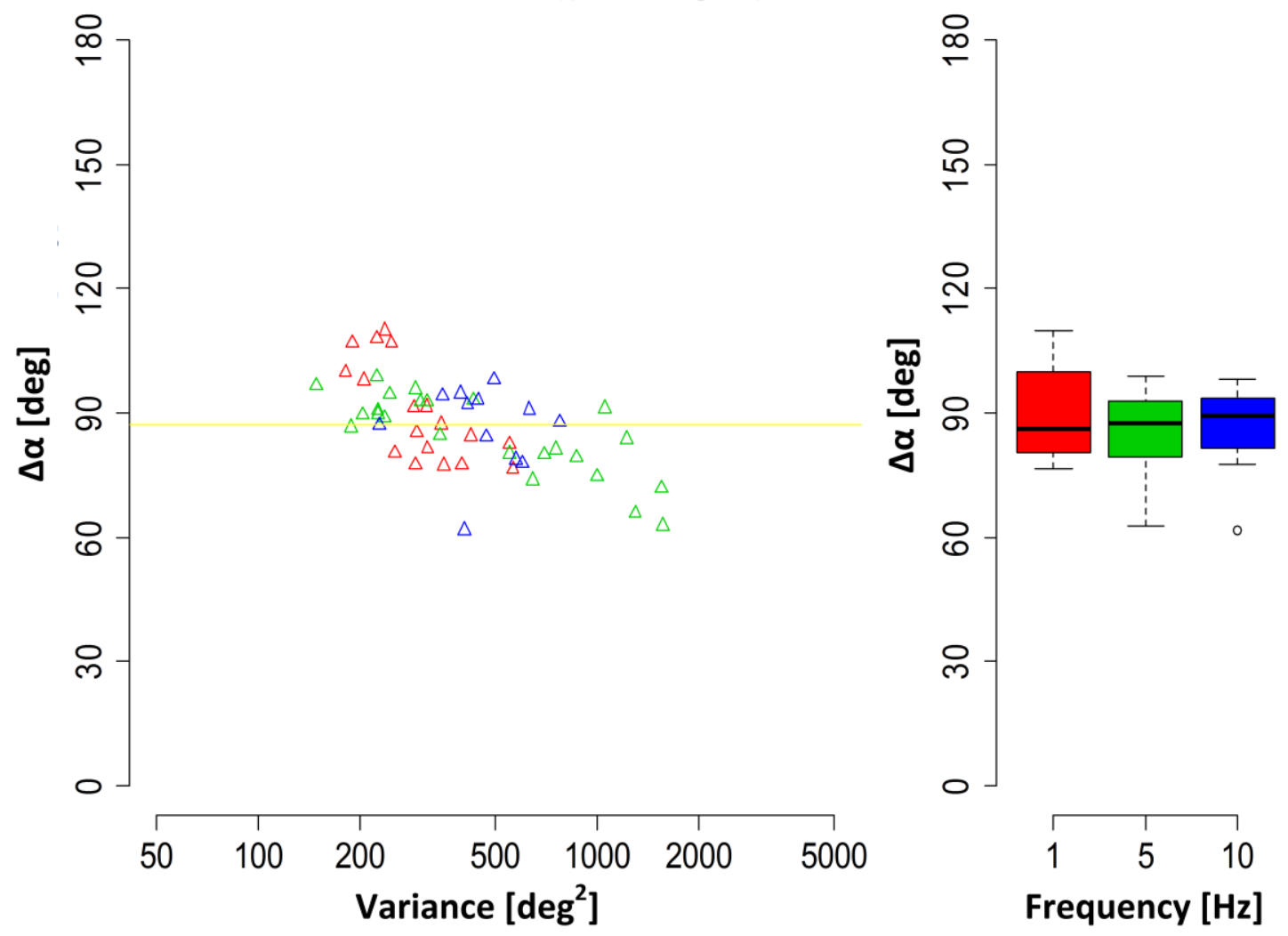
Fibre alignment vs. deflection voltage and frequency

(triangle wave deflection signal)

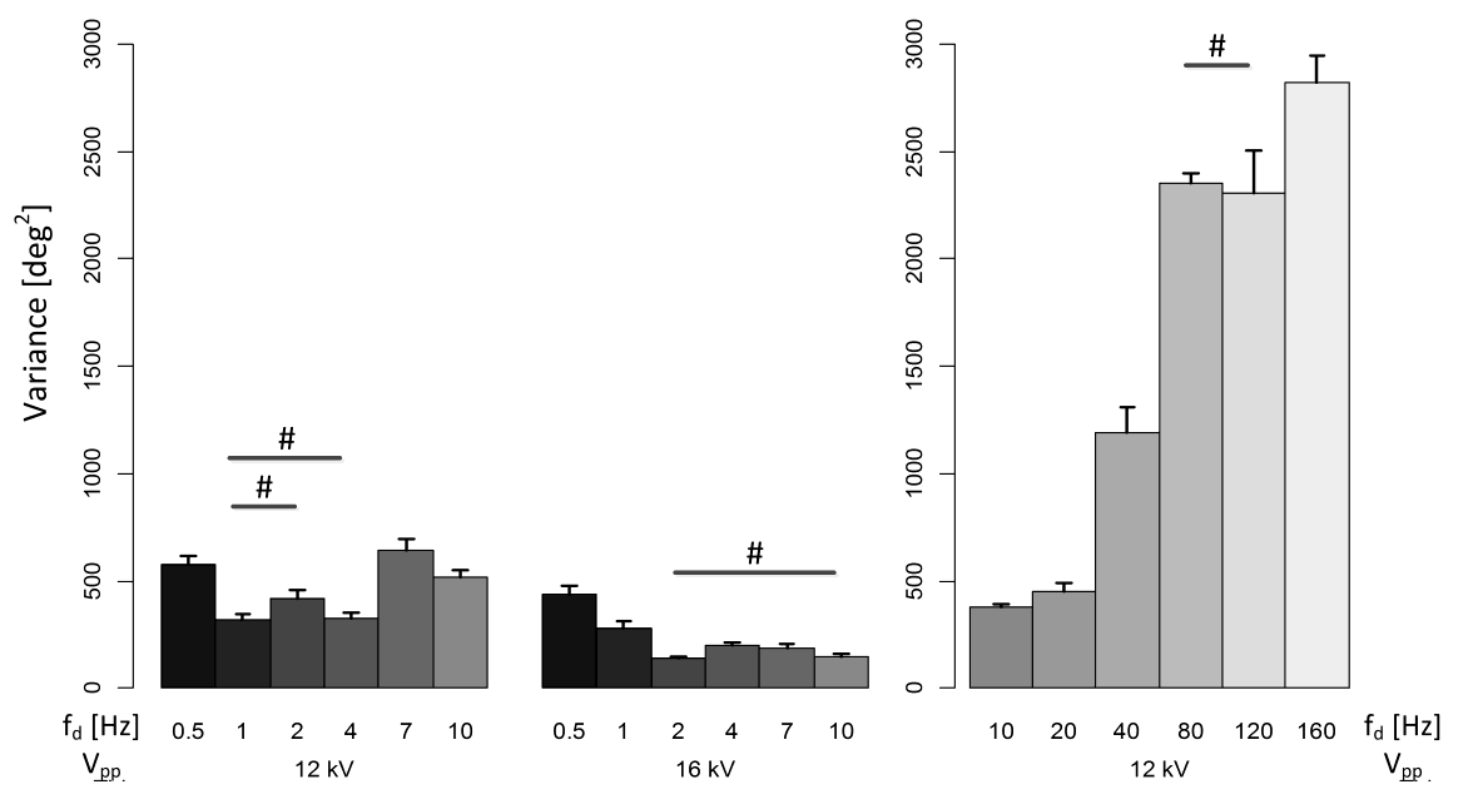


Fibre alignment vs. punch position

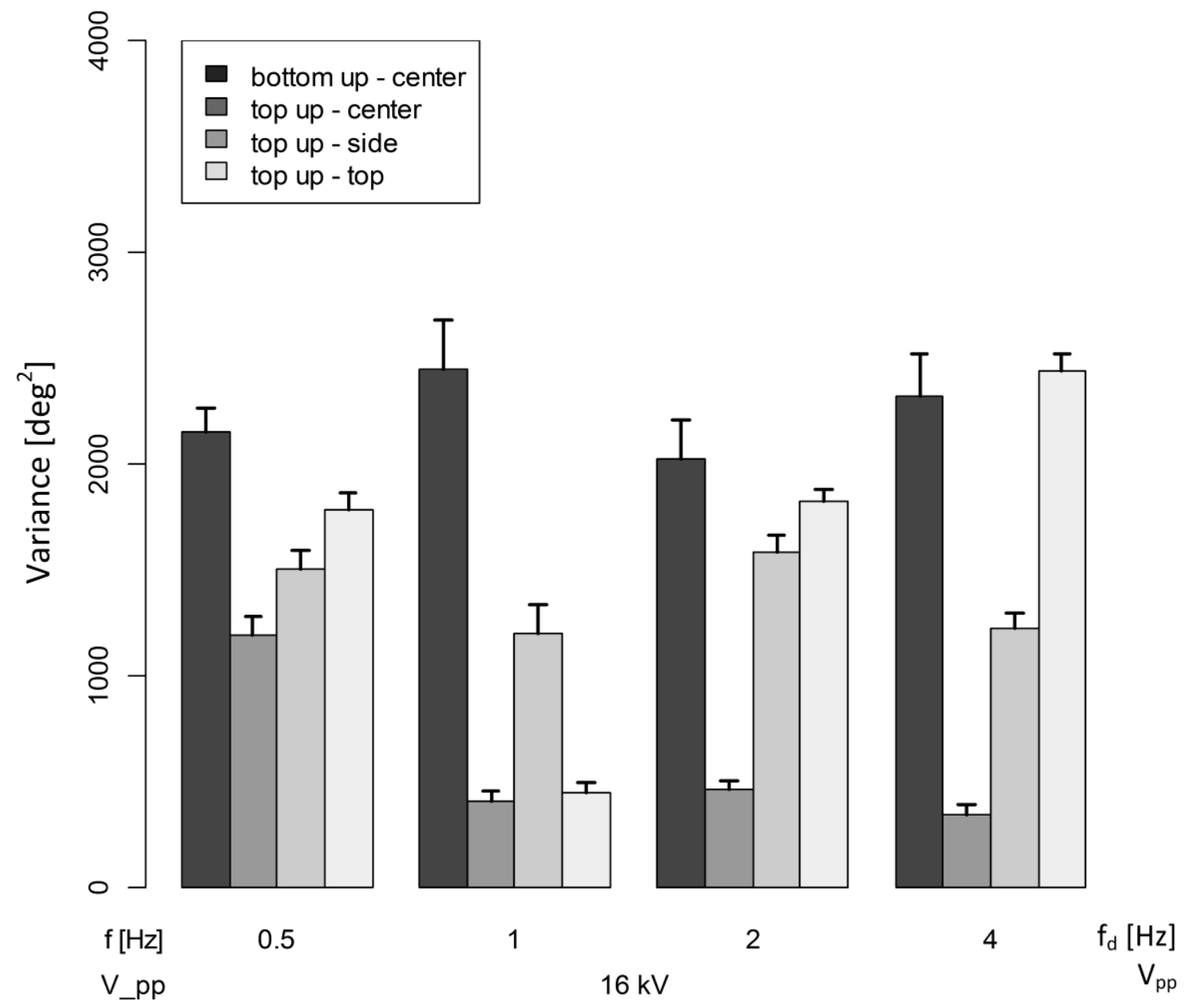




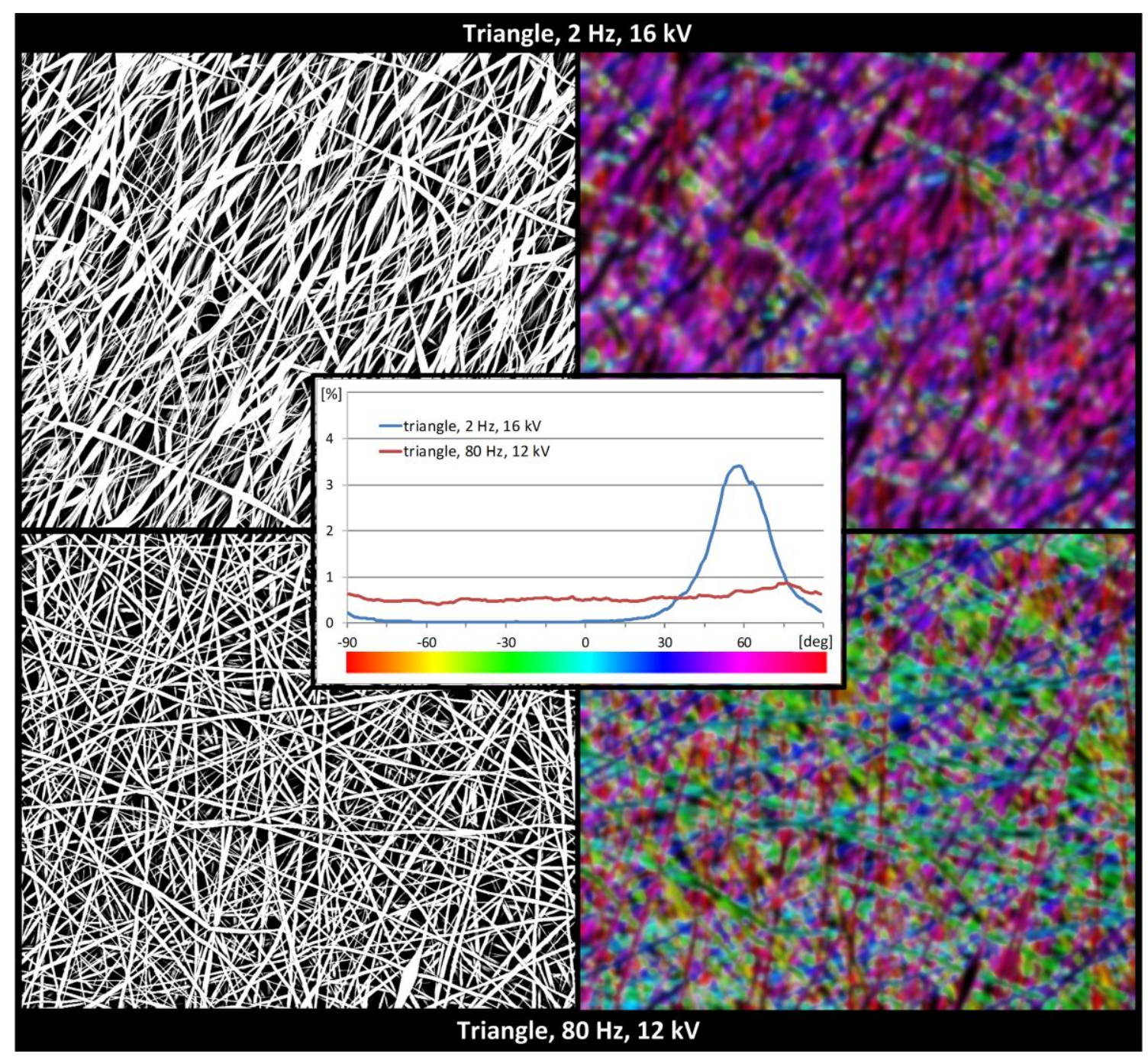


Tensile properties compared to fibre alignment
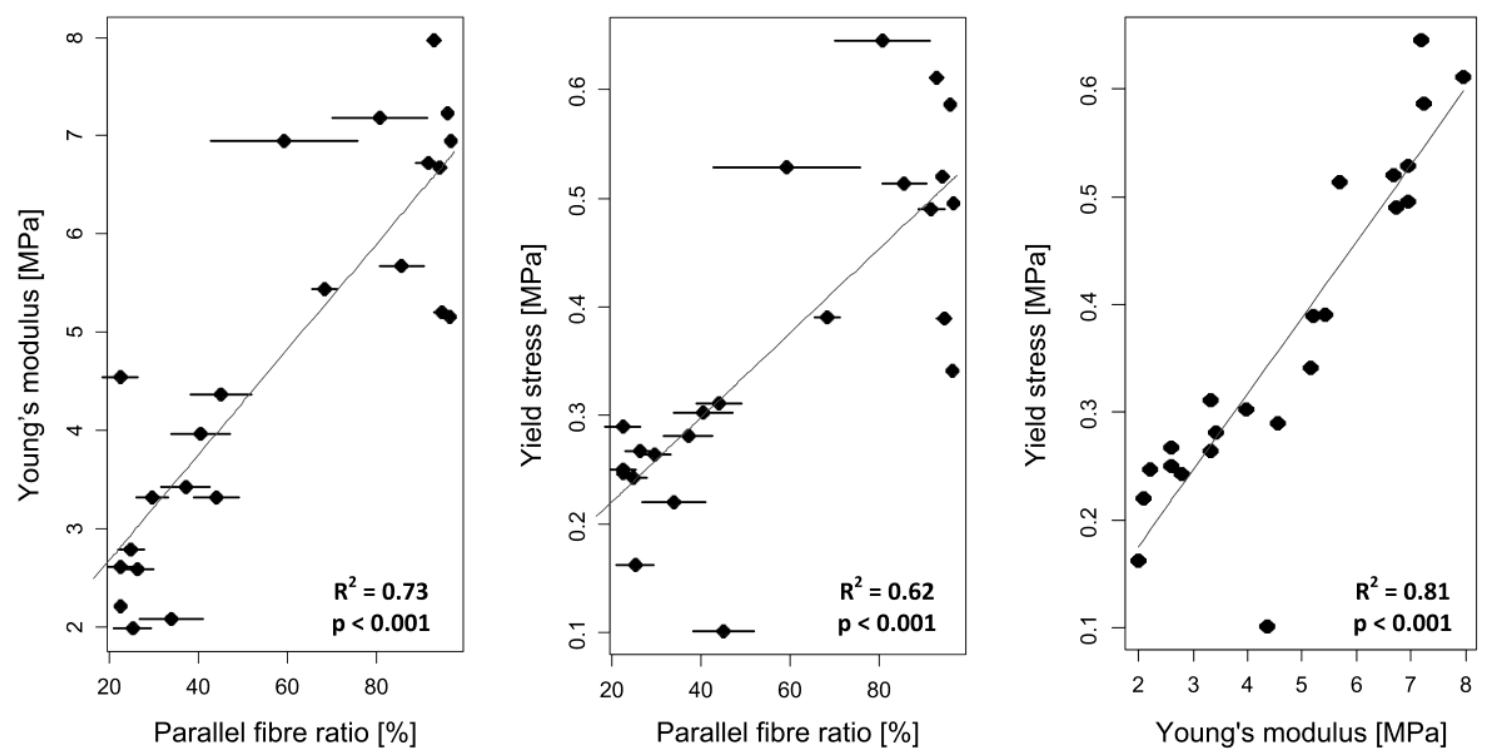


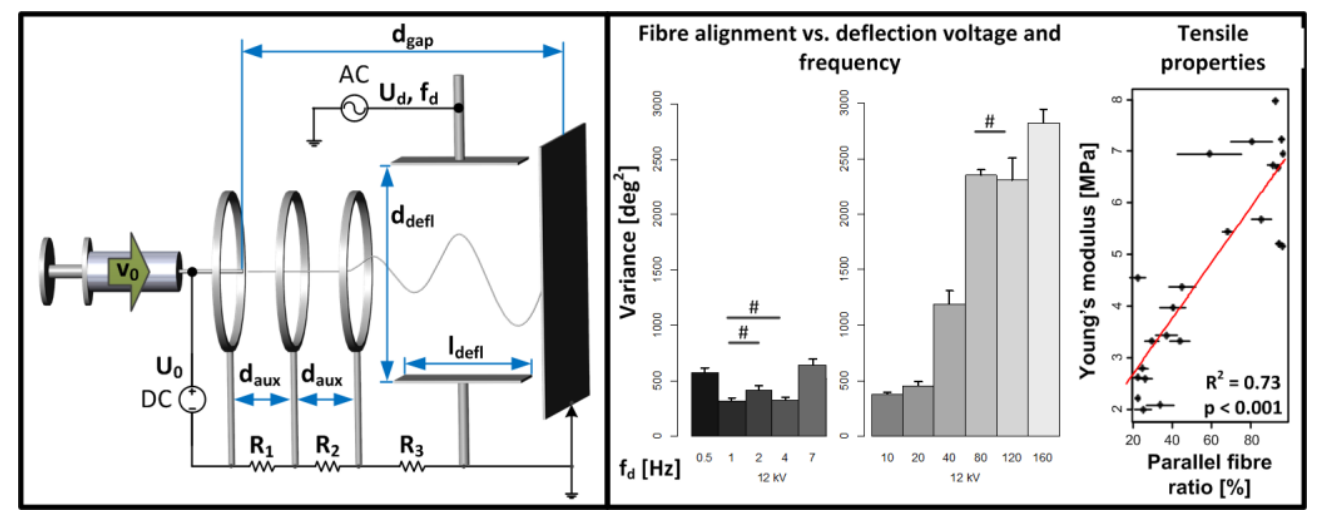


Table 1: Electrospinning parameters investigated. (A) / (B): Deflector potential amplitude 12 kV / 16 kV

\begin{tabular}{|c|c|c|c|c|c|c|c|c|c|c|c|c|c|c|}
\hline $\begin{array}{r}\text { Frequency } \\
{[\mathrm{Hz}]}\end{array}$ & 0.5 & 1 & 2 & 4 & 5 & 7 & 10 & 20 & 40 & 80 & 120 & 160 & 200 & 240 \\
\hline Sine & $-/ X$ & $\begin{array}{l}X / \\
X\end{array}$ & $\begin{array}{l}x / 1 \\
x\end{array}$ & $\begin{array}{l}x / 1 \\
x\end{array}$ & $\begin{array}{l}x / 1 \\
x\end{array}$ & & $\begin{array}{l}x / 1 \\
x\end{array}$ & $\begin{array}{l}-1 \\
x\end{array}$ & & & & & & \\
\hline Triangle & $\begin{array}{l}X / 1 \\
x\end{array}$ & $\begin{array}{l}X / \\
x\end{array}$ & $\begin{array}{l}x / 1 \\
x\end{array}$ & $\begin{array}{l}x / \\
x\end{array}$ & $\begin{array}{l}x / 1 \\
x\end{array}$ & $\begin{array}{l}x / 1 \\
x\end{array}$ & $\begin{array}{l}X / 1 \\
x\end{array}$ & $\begin{array}{l}X / \\
-\end{array}$ & X/ & X/ & $\begin{array}{l}X / \\
-\end{array}$ & $\begin{array}{l}X 1 \\
-\end{array}$ & XI & XI \\
\hline Square & & & & & & & $X /-$ & XI & X/ & XI & XI & XI & XI & XI \\
\hline
\end{tabular}



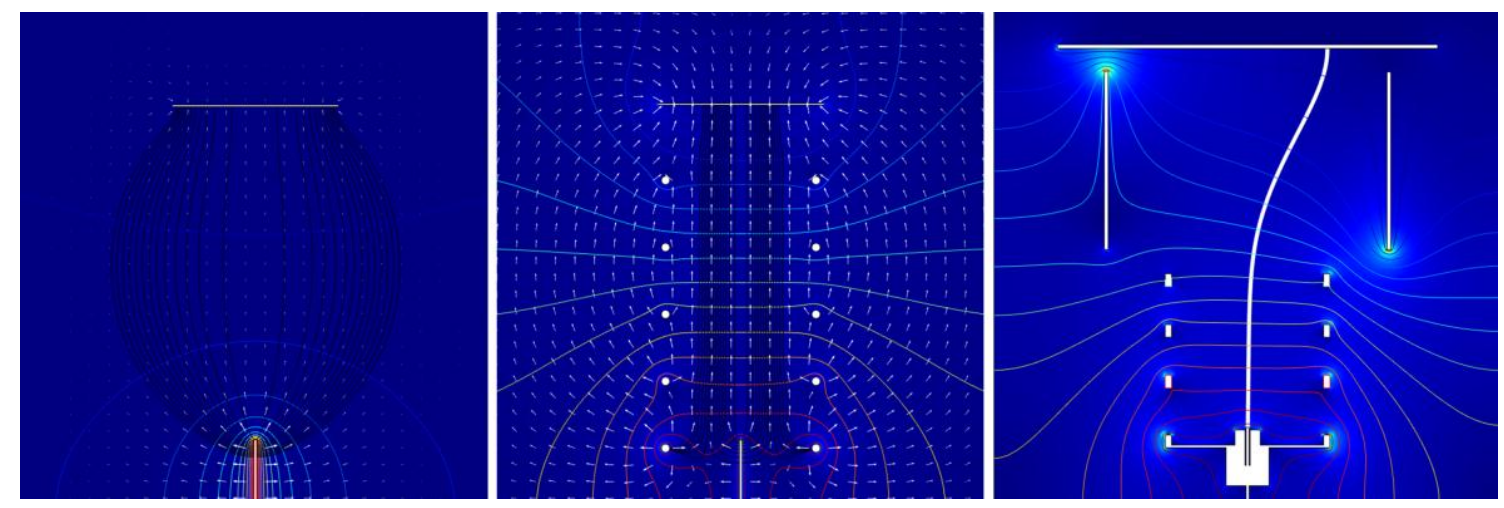
Ratio of aligned fibres vs. variance

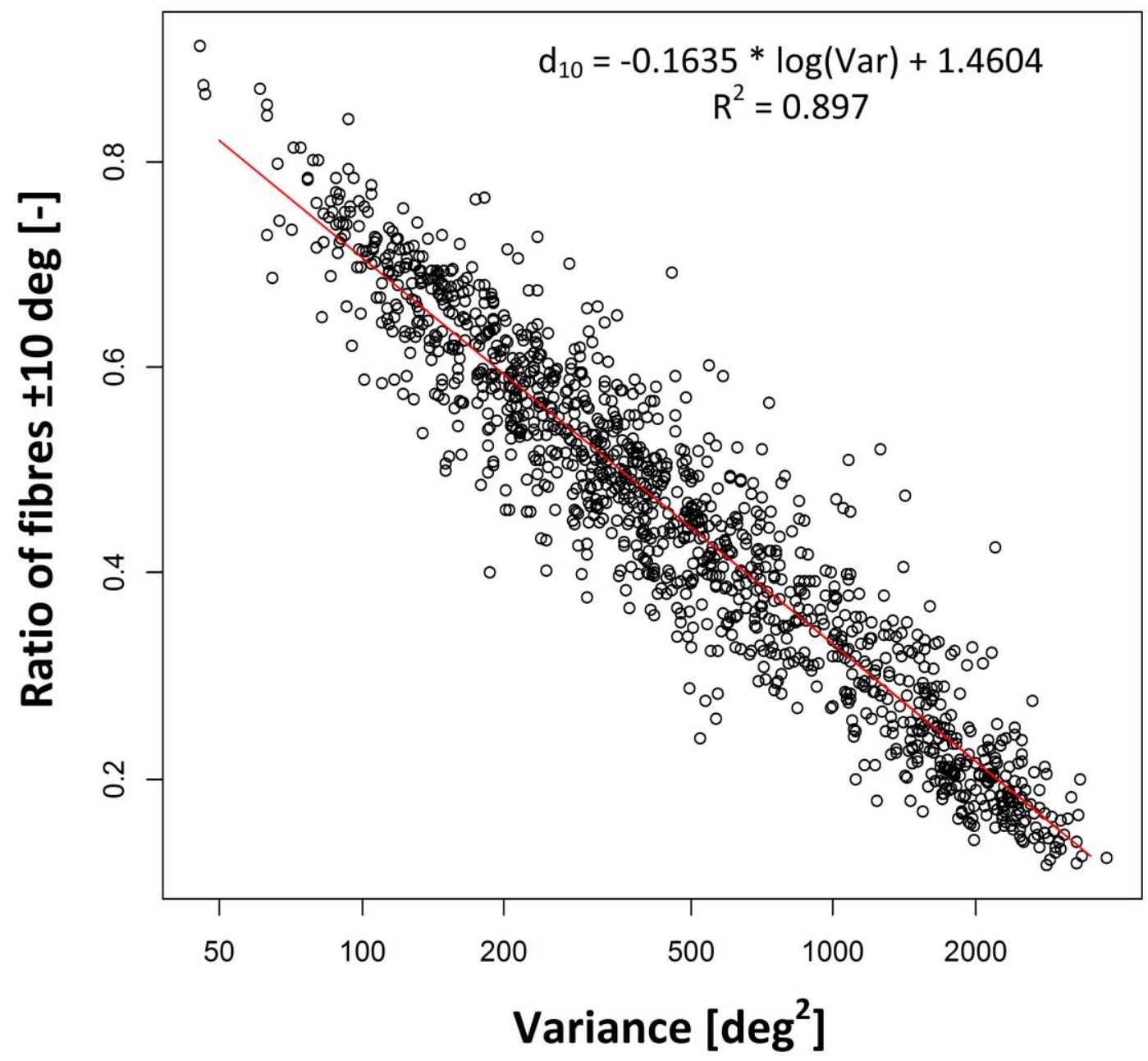


Fibre alignment vs. sine $\&$ triangle wave deflection signal

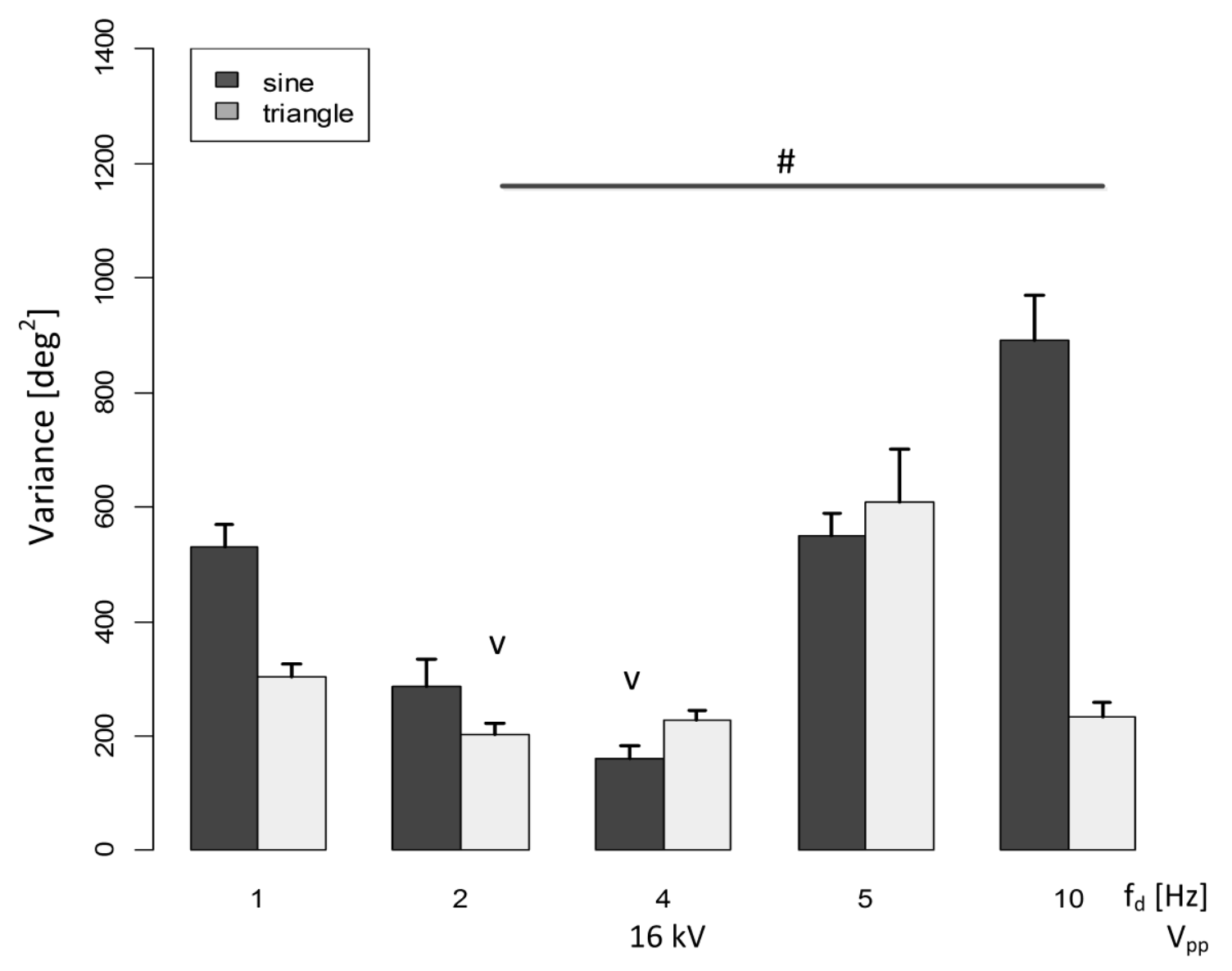


Fibre alignment vs. square \& triangle wave deflection signal

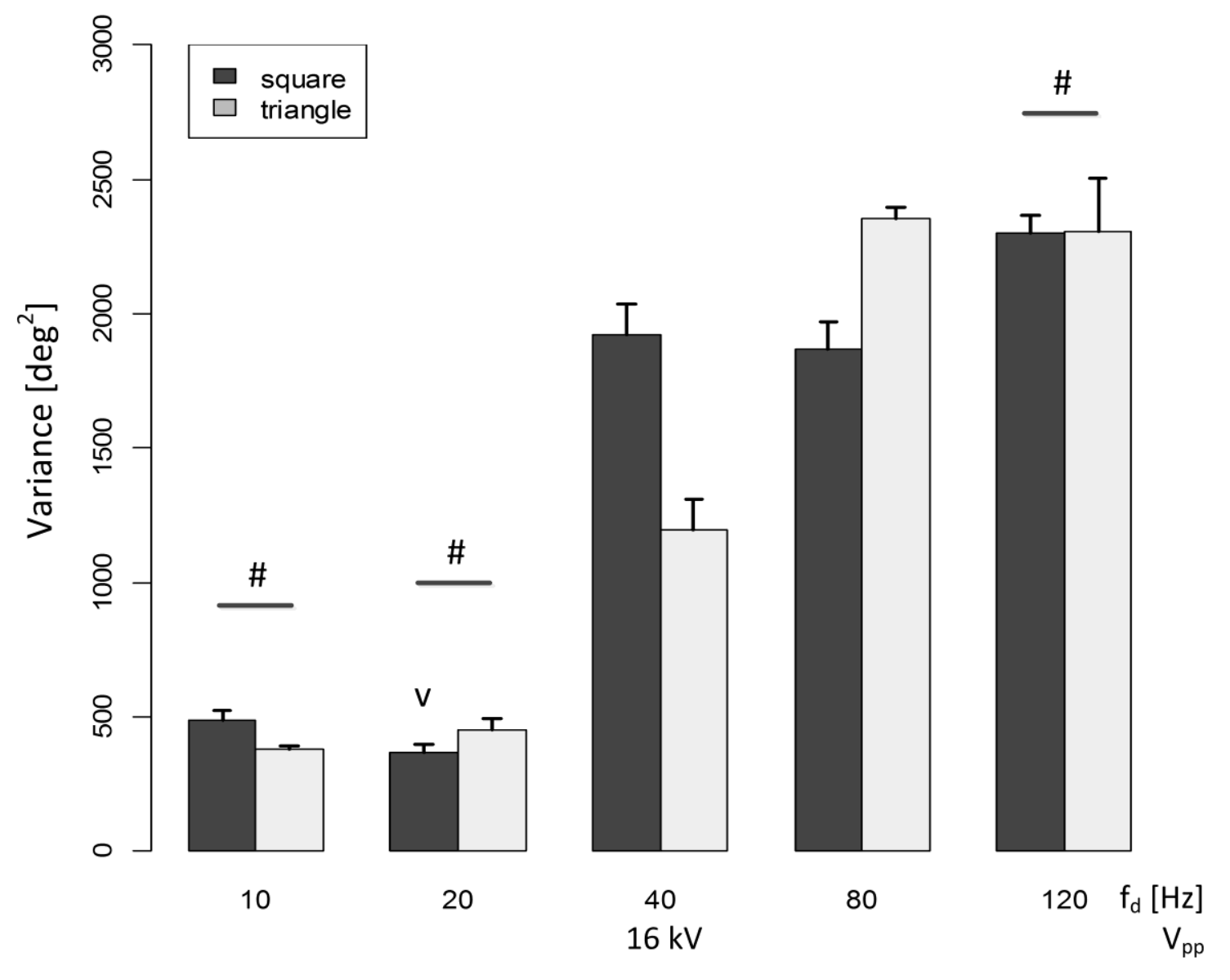

